\title{
On a Construction for Constant-Weight Gray Codes for Local Rank Modulation
}

\author{
Eyal En Gad \\ Elec. Eng. \\ Caltech \\ Pasadena, CA 91125, U.S.A. \\ eengad@caltech.edu
}

\author{
Michael Langberg \\ Comp. Sci. Division \\ Open University of Israel \\ Raanana 43107, Israel \\ mikel@open.ac.il
}

\author{
Moshe Schwartz \\ Elec. and Comp. Eng. \\ Ben-Gurion University \\ Beer Sheva 84105, Israel \\ schwartz@ee.bgu.ac.il
}

\author{
Jehoshua Bruck \\ Elec. Eng. \\ Caltech \\ Pasadena, CA 91125, U.S.A. \\ bruck@paradise.caltech.edu
}

\begin{abstract}
We consider the local rank-modulation scheme in which a sliding window going over a sequence of real-valued variables induces a sequence of permutations. The local rankmodulation, as a generalization of the rank-modulation scheme, has been recently suggested as a way of storing information in flash memory. We study constant-weight Gray codes for the local rank-modulation scheme in order to simulate conventional multilevel flash cells while retaining the benefits of rank modulation. We describe a construction for a codes of rate tending to 1.
\end{abstract}

\section{COnstruction Summary}

It was recently shown in [2] that a local rank modulation scheme may be used to encode information in flash memory cells. Using Gray codes for this scheme enables the use of other rewriting schemes in conjunction with the lower-level Gray code. Though the scheme is defined over permutations, the Gray codes required are equivalent to the following: a sequence of binary vector such that any two adjacent words differ by a single exchange of 10 to 01 (with wrap-around). We provide the first known construction of such codes with rate tending to 1 .

Construction 1. Fix a positive integer $k$. Let $\left\{v_{0}, v_{1}, \ldots, v_{t-1}\right\}$ be a set of $t$ distinct binary vectors of length $n+2$ and weight $w+2$ such that the first and last bit of each $v_{i}$ is 1 . We also require that $k+2 \mid t^{k}$.

The next required ingredient in the construction is a deBruijn sequence of order $k$ over the alphabet $\{0,1, \ldots, t-1\}$. The sequence is of period $t^{k}$ and we denote it by $s_{0}, s_{1}, \ldots, s_{t^{k}-1}$. We remind the reader that windows of size $k$ in the sequence, i.e., $s_{i}, s_{i+1}, \ldots, s_{i+k-1}$, with indices taken modulo $t^{k}$, are all distinct. Such sequences can always be constructed (for example, see [1]).

We now construct the sequence $g_{0}, g_{1}, \ldots, g_{t^{k}-1}$ of $t^{k}$ binary vectors of length $(k+2)(n+2)$ and weight $(k+1)(w+2)$. Each vector is formed by a concatenation of $k+2$ blocks of length $n+2$ in the following way:

$$
\begin{array}{ccccccc}
g_{0}= & v_{s_{k}} & v_{s_{k-1}} & \ldots & v_{s_{1}} & v_{s_{0}} & \mathbf{0} \\
g_{1}= & v_{s_{k}} & v_{s_{k-1}} & \ldots & v_{s_{1}} & \mathbf{0} & v_{s_{k+1}} \\
g_{2}= & v_{s_{k}} & v_{s_{k-1}} & \ldots & \mathbf{0} & v_{s_{k+2}} & v_{s_{k+1}}
\end{array}
$$

This work was supported in part by ISF grant $134 / 10$, ISF grant $480 / 08$, and by internal grant A of the Open University of Israel.

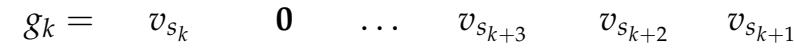

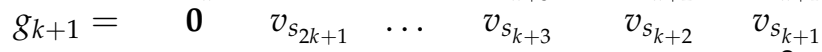

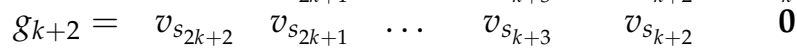

$$
\begin{aligned}
& \begin{array}{lllllll}
g_{k+3}= & v_{s_{2 k+2}} & v_{s_{2 k+1}} & \cdots & v_{s_{k+3}} & \mathbf{0} & v_{s_{2 k+3}}
\end{array} \\
& \begin{array}{lllllll}
g_{k+3} & =v_{S_{2 k+2}} & v_{S_{2 k+1}} & \cdots & \mathbf{0} & v_{S_{2 k+4}} & v_{S_{2 k+3}}
\end{array}
\end{aligned}
$$

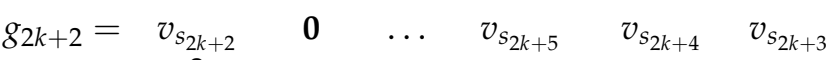

$$
\begin{aligned}
& g_{2 k+3}=\begin{array}{lllllll}
\mathbf{0} & v_{s_{3 k+3}} & \ldots & v_{s_{2 k+5}} & v_{s_{2 k+4}} & v_{s_{2 k+3}}
\end{array} \\
& \begin{array}{lcccccc}
g_{t^{k}-k-2}= & v_{s_{t^{k}-2}} & v_{s_{t^{k}-3}} & \cdots & v_{s_{t^{k}-k-1}} & v_{s_{t^{k}-k-2}} & \mathbf{0} \\
g_{t^{k}-k-1}= & v_{s_{t^{k}-2}} & v_{s_{t^{k}-3}} & \cdots & v_{s_{t^{k}-k-1}} & \mathbf{0} & v_{s_{t^{k}-1}}
\end{array}
\end{aligned}
$$

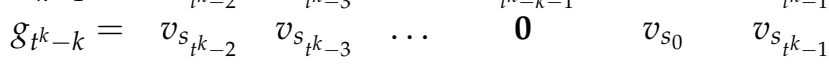

$$
\begin{aligned}
& \begin{array}{lllllll}
g_{t^{k}-2} & =v_{s_{t^{k}-2}} & \mathbf{0} & \ldots & v_{s_{1}} & v_{s_{0}} & v_{s_{t^{k}-1}}
\end{array} \\
& g_{t^{k}-1}=\begin{array}{lllllll}
\mathbf{0} & v_{s_{k-1}} & \cdots & v_{s_{1}} & v_{s_{0}} & v_{s_{t}-1}
\end{array}
\end{aligned}
$$

where 0 denotes the all-zero vector of length $n+2$.

We call $g_{0}, g_{1}, \ldots, g_{t^{k}-1}$ the anchor vectors. Between any two anchors, $g_{i}$ and $g_{i+1}$, a sequence of vectors called auxiliary vectors and denoted $g_{i}^{0}, g_{i}^{1}, \ldots, g_{i}^{\ell_{i}}$, is formed in the following way: The only allowed transition is a 10 changed into a 01 . First the rightmost 1 in the block $v_{s_{i}}$ is moved to the right, step by step, to the position of the rightmost 1 in $v_{s_{i+k+1}}$. The process then repeats with a sequence of transitions moving the secondfrom-right 1 in $v_{s_{i}}$ to the position of the second-from-right 1 in $v_{s_{i+k+1}}$, and so on, until $v_{s_{i}}$ is moved one block to the right and changed into $v_{s_{i+k+1}}$. The resulting list of anchor vectors and, in between them, auxiliary vectors, is the constructed code.

Theorem 1. The sequence constructed in Construction 1 is a constant-weight local rank modulation Gray code of size $N$ which satisfies $t^{k}(w+2) \leqslant N \leqslant t^{k}(w+2)(n+2)$.

Corollary 2. There exists an infinite family $\left\{G_{i}\right\}_{i=1}^{\infty}$ of cyclic Gray codes as above, $n_{i+1}>n_{i}$, for which $\lim _{i \rightarrow \infty} \mathrm{R}\left(G_{i}\right)=1$.

\section{REFERENCES}

[1] S. W. Golomb, Shift Register Sequences. Holden-Day, San Francisco, 1967.

[2] M. Schwartz, "Constant-weight Gray codes for local rank modulation," in Proceedings of the 2010 IEEE International Symposium on Information Theory (ISIT2010), Austin, TX, U.S.A., Jun. 2010, pp. 869-873. 